\title{
Impact of sensor and measurement timing errors on model- based insulin sensitivity
}

\begin{tabular}{|c|c|}
\hline Christopher G. Pretty ${ }^{1}$ & c.pretty@ulg.ac.be \\
\hline Matthew Signal2 & matthew.signal@pg.canterbury.ac.nz \\
\hline Liam Fisk ${ }^{2}$ & liam.fisk@pg.canterbury.ac.nz \\
\hline Sophie Penning1 & sophie.penning@ulg.ac.be \\
\hline Aaron Le Compte ${ }^{2}$ & aaron.lecompte@canterbury.ac.nz \\
\hline Geoffrey M. Shaw ${ }^{3}$ & geoff.shaw@cdhb.govt.nz \\
\hline Thomas Desaive $^{1}$ & tdesaive@ulg.ac.be \\
\hline J. Geoffrey Chase ${ }^{2}$ & geoff.chase@canterbury.ac.nz \\
\hline
\end{tabular}

1. University of Liege, GIGA Cardiovascular Sciences, Liege, Belgium

2.University of Canterbury, Centre for Bioengineering, Christchurch, New Zealand

3.Christchurch Hospital, Department of Intensive Care, Christchurch, New Zealand

Keywords: Biomedical system modelling; simulation and visualization; Control of physiological and clinical variables; 


\section{Abstract}

A model-based insulin sensitivity parameter $(S I)$ is often used in glucose-insulin system models to define the glycaemic response to insulin. As a parameter identified from clinical data, insulin sensitivity can be affected by blood glucose (BG) sensor error and measurement timing error, which can subsequently impact analyses or glycaemic variability during control. This study assessed the impact of both measurement timing and BG sensor errors on identified values of $S I$ and its hour-to-hour variability within a common type of glucose-insulin system model.

Retrospective clinical data were used from 270 patients admitted to the Christchurch Hospital ICU between 2005 and 2007 to identify insulin sensitivity profiles. We developed error models for the Abbott Optium Xceed glucometer and measurment timing from clinical data. The effect of these errors on the reidentified insulin sensitivity was investigated by Monte-Carlo analysis.

The results of the study show that timing errors in isolation have little clinically significant impact on identified $S I$ level or variability. The clinical impact of changes to $S I$ level induced by combined sensor and timing errors is likely to be significant during glycaemic control. Identified values of $S I$ were mostly ( $90^{\text {th }}$ percentile) within $29 \%$ of the true value when influenced by both sources of error. However, these effects may be overshadowed by physiological factors arising from the critical condition of the patients or other under-modelled or unmodelled dynamics. Thus, glycaemic control protocols that are designed to work with data from glucometers need to be robust to these errors and not be too aggressive in dosing insulin. 


\section{Introduction}

Physiological glucose-insulin system models typically rely on some form of insulin sensitivity parameter to characterise the patient-specific glycaemic response to exogenous insulin $[1,2]$. This model-based insulin sensitivity parameter (SI) is identified for some period of time using blood glucose (BG) concentration and insulin and nutrition administration data. Errors in blood glucose concentration and measurement timing can thus affect the identified values of $S I$.

In the busy intensive care unit (ICU) environment, BG measurements are rarely taken and recorded at an exact, scheduled time. Sensor errors add uncertainty to the measured BG concentration. Both errors propagate through to SI during parameter identification, which in turn may impact subsequent analyses or glycaemic variability during control.

Typical point-of-care glucometers claim to have measurement errors in the range $2-10 \%$ [3-7]. The uncertainty in BG concentration resulting from sensor error impacts the identified values of SI through altering the glucose flux that must be balanced by the insulin-mediated glucose disposal term in the glucoseinsulin system model.

The objective of this investigation was to assess the impact of both measurement timing and sensor errors on identified values of SI and its hour-to-hour variability. Specifically, the SI parameter from a glucose-insulin system model similar to that described by Lin et al. [1] was investigated with patient data from the Christchurch Hospital ICU. 


\section{Subjects and Methods}

\subsection{Patients}

This study was conducted as retrospective analyses of data from 270 patients admitted to the Christchurch Hospital ICU between 2005 and 2007. All patients were on the SPRINT protocol for at least 24 hours [8]. Table 1 shows a summary of the cohort details. The Upper South Regional Ethics Committee, New Zealand granted approval for the audit, analysis and publication of this data.

Table 1. Cohort details summary. Data are shown as median [interquartile range] where appropriate

\begin{tabular}{|l|c|}
\hline $\mathrm{N}$ & 270 \\
\hline Age (years) & 65 [49-73] \\
\hline Gender (M/F) & $165 / 105$ \\
\hline Operative/Non-Operative & $104 / 166$ \\
\hline Hospital mortality (\%) & $27 \%$ \\
\hline APACHE II score & $19[16-25]$ \\
\hline APACHE II ROD (\%) & $30[17-53]$ \\
\hline Diabetic status (T1DM/T2DM) & $10 / 34$ \\
\hline ICU length of stay (hrs) & $160[77-346]$ \\
\hline
\end{tabular}

\subsection{Model-based insulin sensitivity}

The glucose-insulin system model used in this study was an enhanced version of the ICING model described by Lin et al. [1], with a new endogenous insulin secretion sub-model (7) derived from data not yet published. The model is defined below in Equations (1)-(7). Model parameters, rates and constants were generally as described in Lin et al. [1], except for $n_{I}, n_{C}$ and $V_{I}$ which have been adjusted to $0.006 \mathrm{~min}^{-1}, 0.006 \mathrm{~min}^{-1}$ and $4.0 \mathrm{~L}$, respectively. These changes were made based on an analysis of results from several microdialysis studies and the population parameters from Van Cauter et al. [9].

Endogenous insulin secretion was modelled as a function of BG. Sub-model parameters, $u_{\min }$ and $u_{\max }$ are $16.7 \mathrm{mU} \cdot \mathrm{min}^{-1}$ and $266.7 \mathrm{mU} \cdot \mathrm{min}^{-1}$, respectively. 
For non-diabetic patients, $k_{1}$ and $k_{2}$ take the values 14.9 mU.L.mmol-1. $\mathrm{min}^{-1}$ and 49.9 mU.min-1.

The model was implemented in MATLAB (2012a, Natick, MA), and a value of SI identified each hour for every patient using clinical BG, insulin and nutrition records. The parameter identification was performed using an integral method that ensured the global optimum value was located [10].

$$
\begin{aligned}
& \dot{G}(t)=-p_{G} G(t)-\text { SI. } G(t) \frac{Q(t)}{1+\alpha_{G} Q(t)}+\frac{P(t)+E G P-C N S}{V_{G}} \\
& \dot{Q}(t)=n_{I}(I(t)-Q(t))-n_{C} \frac{Q(t)}{1+\alpha_{G} Q(t)} \\
& \dot{I}(t)=-n_{K} I(t)-n_{L} \frac{I(t)}{1+\alpha_{I} I(t)}-n_{I}(I(t)-Q(t))+\frac{u_{e x}(t)}{V_{I}}+\left(1-x_{L}\right) \frac{u_{e n}(G)}{V_{I}} \\
& P(t)=\min \left(d_{2} P 2, P_{\max }\right)+P N(t) \\
& \dot{P} 1(t)=-d_{1} P 1+D(t) \\
& \dot{P} 2(t)=-\min \left(d_{2} P 2, P_{\max }\right)+d_{1} P 1 \\
& u_{e n}(G)=\min \left(\max \left(u_{\min }, k_{1} G(t)+k_{2}\right), u_{\max }\right)
\end{aligned}
$$

The model-based insulin sensitivity parameter has been shown to correlate well with the insulin sensitivity index (ISI) determined by the gold-standard euglycaemic-hyperinsulinaemic clamp ( $\mathrm{r}>0.90)$ [11]. Hour-to-hour SI variability is defined in (8).

$\Delta \% S I_{k}=100 \times \frac{\left(S I_{k+1}-S I_{k}\right)}{S I_{k}}$

\subsection{Blood glucose sensor error}

This study uses preliminary information from an on-going investigation evaluating the performance of several different point-of-care glucometers in the Christchurch Hospital ICU. For the purposes of this study, an error model was derived for the Abbott Optium Xceed glucometer (Abbott Diabetes Care, Alameda, CA), a commonly available, inexpensive device that measures the glucose concentration in a whole blood sample, and reports a plasma equivalent 
glucose concentration. Test strips used in this study were validated for a haematocrit range of $20-70 \%$ [12].

The study in Christchurch used arterial blood samples, each drawn by an experience intensive care nurse. The glucose concentration of each sample was determined using a Radiometer ABL90 Flex blood gas analyser (BGA) (Copenhagen, Denmark) and simultaneously with up to 5 separate glucometers. However, due to device error, availability, or clinical reasons, some samples were tested on fewer than 5 glucometers. All measurements were completed within 5 minutes of the blood draw. Data from 242 samples (758 glucometer measurements) were available to derive the error model from 758 paired measurements.

Whole blood glucose results from the BGA were adjusted with the measured haematocrit [13] to yield plasma glucose concentrations for direct comparison with the glucometers. Figure 1 shows scatter and Bland-Altman plots of the paired data and Figure 2 shows the kernel density model of the glucometer BG values given the BGA values.
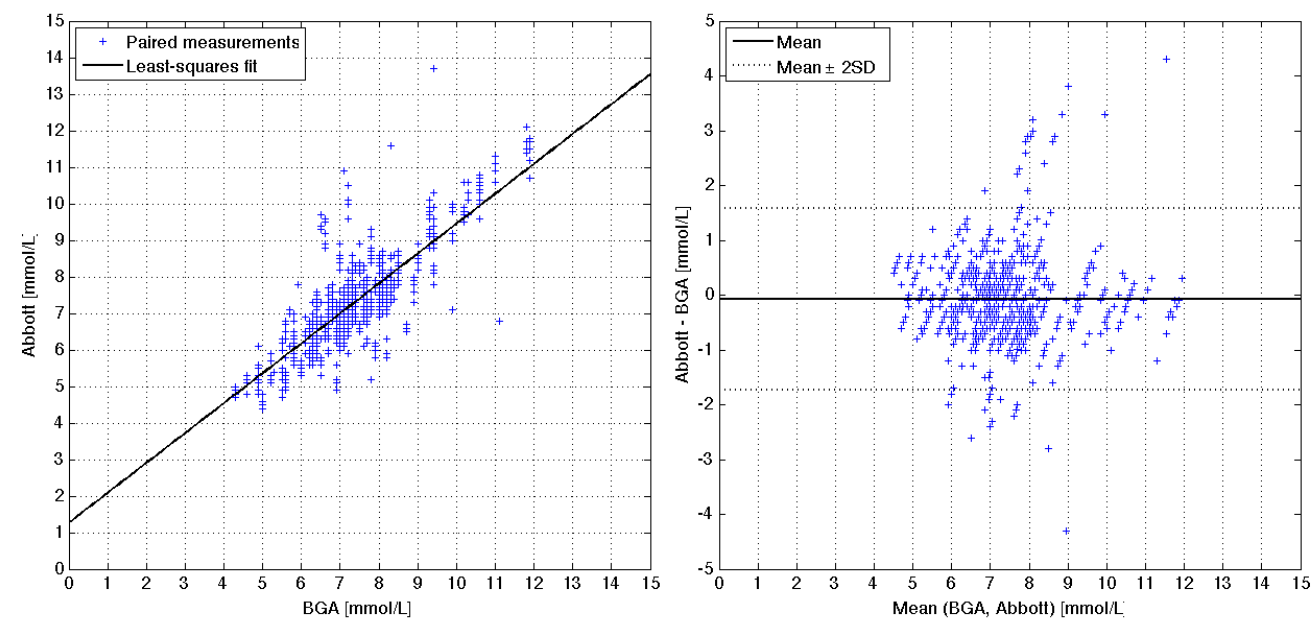

Figure 1. Scatter (left) and Bland-Altman (right) plots of Abbott Optium Xceed glucometer performance against reference Radiometer ABL90 Flex blood gas analyser measurements. 


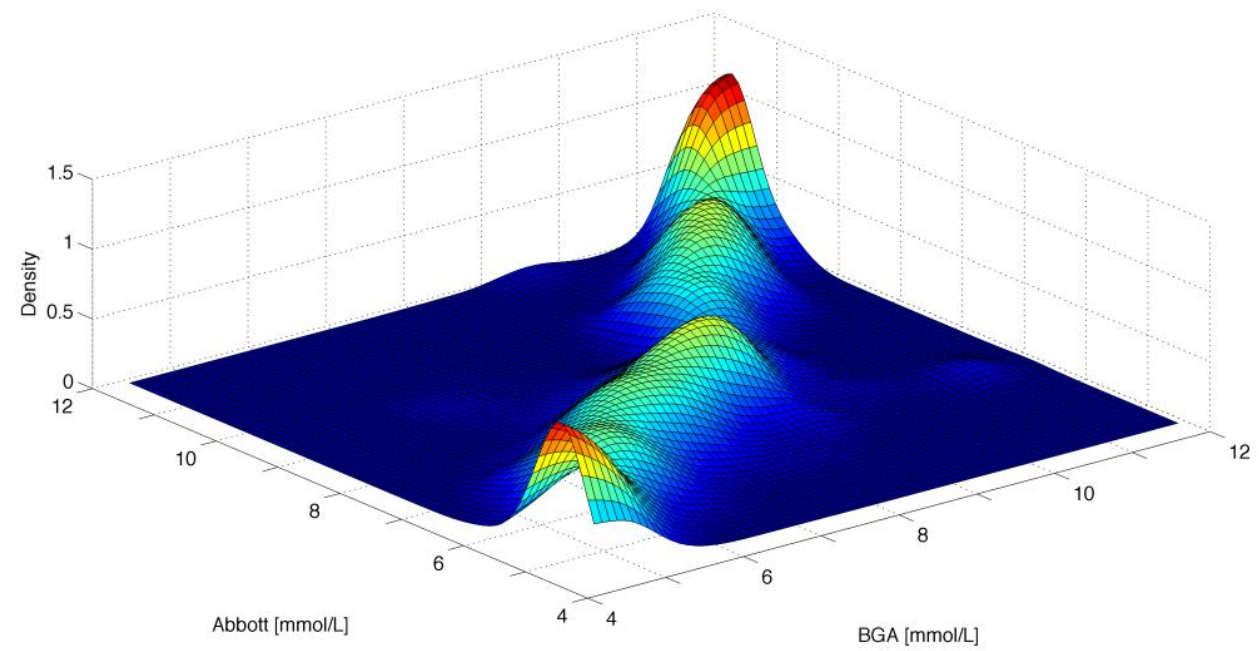

Figure 2. Kernel density model of Abbott Optium Xceed glucometer performance based on 758 paired measurments with a blood gas analyser (BGA)

Table 2 shows a simplified, binned error model derived from the paired data, for comparison with the published errors (from test-strip packet insert) presented in Table 3 [12]. An estimate of the sensor bias was derived from the correlation data. Precision was modelled using the reported standard deviations, linearly interpolated within the reported range and held constant outside.

Table 2. Binned errors for the Abbott Optium Xceed glucometer based on paired measurements from Christchurch Hospital ICU.

\begin{tabular}{|l|c|c|c|c|c|}
\hline $\begin{array}{l}\text { Reference BG } \\
(\mathrm{mmol} / \mathrm{L})\end{array}$ & $<5.9$ & $6.0-6.9$ & $7.0-7.9$ & $8.0-8.9$ & $>9.0$ \\
\hline $\begin{array}{l}\text { Number of } \\
\text { measurements }\end{array}$ & 88 & 180 & 261 & 156 & 73 \\
\hline $\begin{array}{l}\text { Error mean } \\
\text { (mmol/L) }\end{array}$ & 0.28 & 0.19 & -0.05 & -0.56 & -0.26 \\
\hline $\begin{array}{l}\text { Error std. dev. } \\
\text { (mmol/L) }\end{array}$ & 0.55 & 0.84 & 0.83 & 0.63 & 0.91 \\
\hline
\end{tabular}


Table 3. Error model for the Abbott Optium Xceed glucometer based on data published by the manufacturer [12].

\begin{tabular}{|l|c|c|c|c|c|c|}
\hline $\begin{array}{l}\text { Reference BG } \\
(\mathrm{mmol} / \mathrm{L})\end{array}$ & $<2.4$ & 2.4 & 5.6 & 8.0 & 20 & $>20$ \\
\hline $\begin{array}{l}\text { Error mean } \\
(\mathrm{mmol} / \mathrm{L})\end{array}$ & +0.25 & +0.25 & +0.19 & +0.14 & -0.10 & -0.10 \\
\hline $\begin{array}{l}\text { Error std. dev. } \\
\text { (mmol/L) }\end{array}$ & 0.13 & 0.13 & 0.22 & 0.30 & 0.94 & 0.94 \\
\hline
\end{tabular}

\subsection{Timing error}

Measurements and interventions during the SPRINT protocol were 1 or 2-hourly and intended to be taken on the hour. These measurements were recorded by hand and attributed to the nearest hour on the standard paper 24-hour charts used in the Christchurch Hospital ICU. Hence, any discrepancies between the actual measurement time and the 'nearest hour' were lost.

Recent pilot trials of the STAR (Stochastic TARgeted) protocol at Christchurch Hospital ICU [14] provide data to generate a timing error model (1651 measurements on 20 patients). The STAR protocol is implemented on a tablet computer, thus the exact time when BG measurements were entered was recorded and can be compared to the written records. Using the discrepancies between scheduled and actual BG measurements, a model of timing error can be generated and applied to data from the SPRINT protocol. Although the STAR protocol differs from SPRINT, particularly with its computerised interface, it was used by the same clinical staff in the same unit. Hence, it may be assumed that timing errors in making measurements will be similar.

Timing errors were limited to a maximum of 28 minutes for this analysis. Errors of \pm 30 minutes or more are considered as missed or additional treatments as they are closer to the next hour than the previous. Both these cases relate to protocol compliance and are thus are not considered as timing errors for the purposes of this analysis. $92 \%$ of recorded timing errors fell within the range -28 to 28 minutes. 
The empirical timing error distributions are shown in Figure 3. Errors from these distributions were applied additively to the SPRINT data by randomly sampling from the error vectors. The errors were applied to both the measurement and intervention timing. Thus, the measurements and interventions remained synchronised, as they would in the hospital.
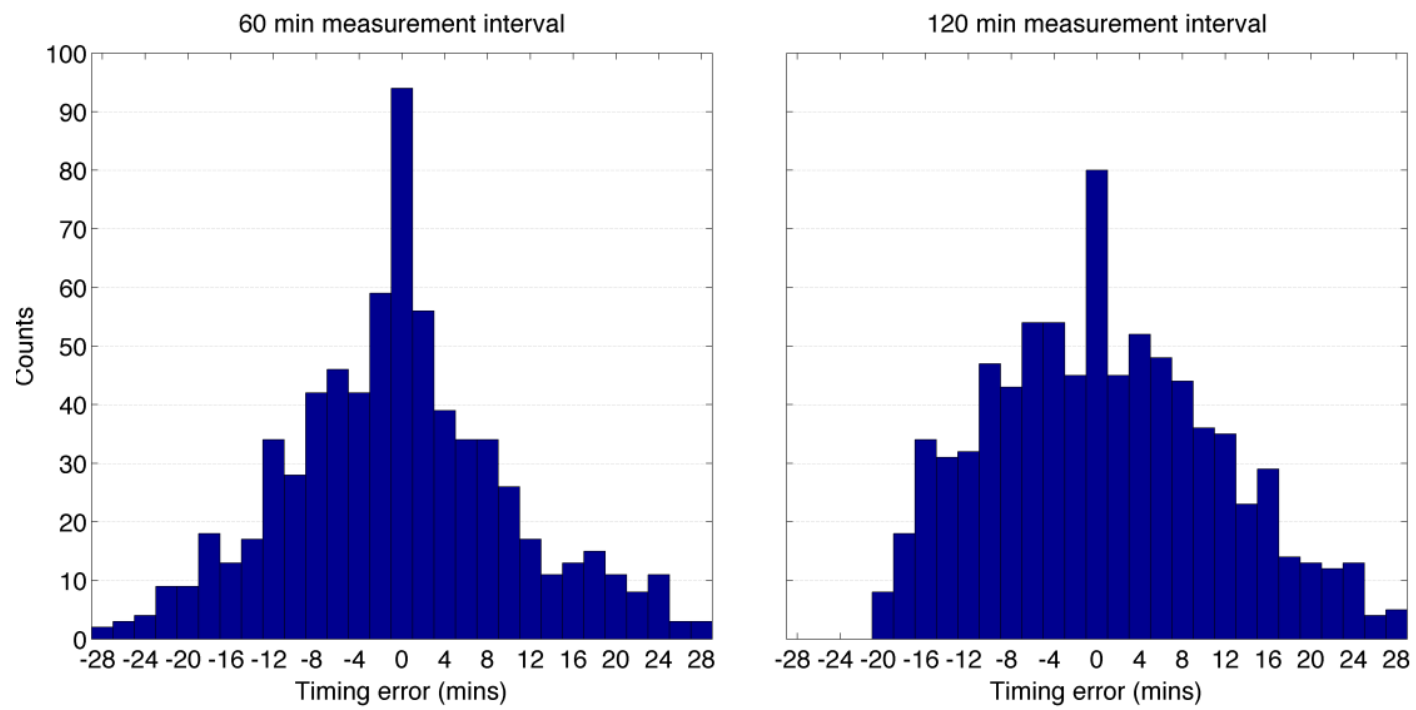

Figure 3. Timing error models based on data from the STAR pilot trials [14]. Errors from 1- and 2 hour measurements are shown on the left and right, respectively.

\subsection{Analysis}

To assess the effects of random timing and sensor errors on SI, Monte Carlo simulations were performed. Due to the non-linearity of the model equations and the numerical identification of SI, an analytical derivation of these effects was not possible.

For the Monte Carlo simulations, the SI profile of each patient in the cohort was refitted $n=50$ times with randomly sampled errors from applied to the observed timing and BG concentrations. The SI profiles identified without additional random errors were considered the 'true' profiles, and the Monte Carlo profiles were compared to these to assess the impact each of the sources of error. Comparisons of both $S I$ level and hour-to-hour variability were made. 
BG errors were sampled from the kernel density model, within the range 4.4$11.9 \mathrm{mmol} / \mathrm{L}$ in which paired data existed. Outside this range, errors were assumed to be normally distributed with parameters shown in Table 3, from the published error model.

To facilitate comparisons when timing errors were applied, SI was identified in 60-minute intervals, rather than between BG measurements. This use of fixed, 60-minute fitting intervals and linear interpolation between measurements is consistent with the methodology used for glycaemic control by the STAR protocol.

To analyse the impact of errors on the identified SI level, the variation induced by the simulated errors at each hour was assessed across the Monte Carlo simulations. Figure 3 illustrates the methodology for SI level comparisons between the $n$ Monte Carlo simulations and the true data.

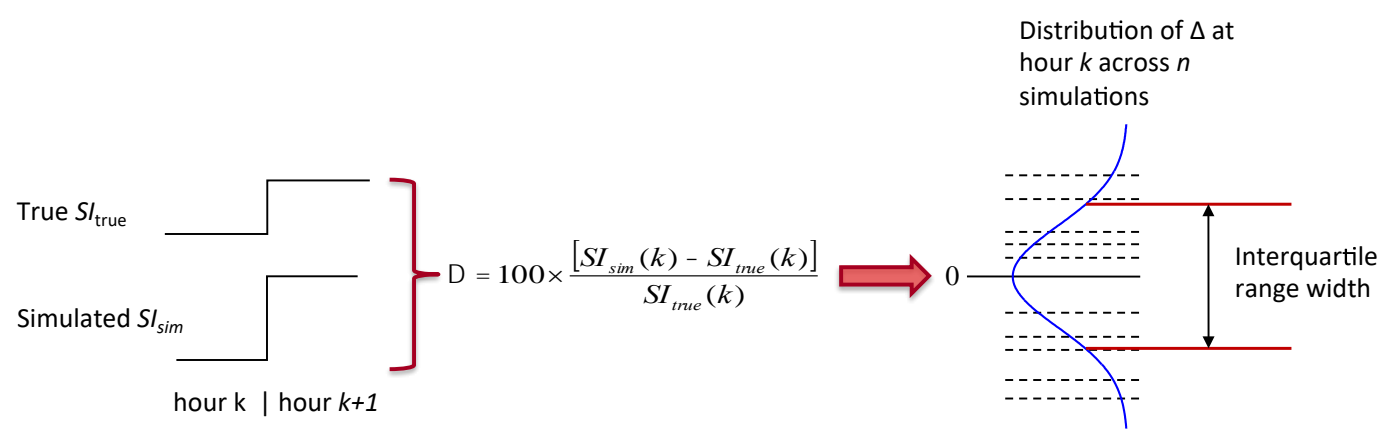

Figure 4. SI level comparison method for the Monte Carlo simulations with added sensor and timing error. The width of the interquartile range of differences was used to characterise the variability in level induced by the errors.

This analysis method results in one interquartile range (IQR) width value per patient hour. For the 270 patient SPRINT cohort, there were approximately 43,000 hours of data. These 43,000 IQR widths were presented as cumulative distributions to show the overall effect of the errors on the cohort.

The hour-to-hour variability (\%) of simulated data could not be compared to the true variability using ratios as the distribution of true variability was centred close to zero, causing ratios to take extreme values. Thus, the impact of errors on 
hour-to-hour variability can be quantified by the width of the IQR across the simulations at each hour. 


\section{Results and Discussion}

\subsection{Timing error}

Figure 5 shows the impact of timing errors on identified SI level (left panel) and variability (right panel). For $90 \%$ of hours, the IQR width of SI level was $10 \%$. Thus, for those 38,700 hours, half the simulations resulted in SI values within $\pm 5 \%$ of the true value, in the case of a symmetrical distribution. Similarly, for variability, 90\% of hours had an IQR width of hour-to-hour changes of less than $14 \%$, or $\pm 7 \%$ about the simulation median.
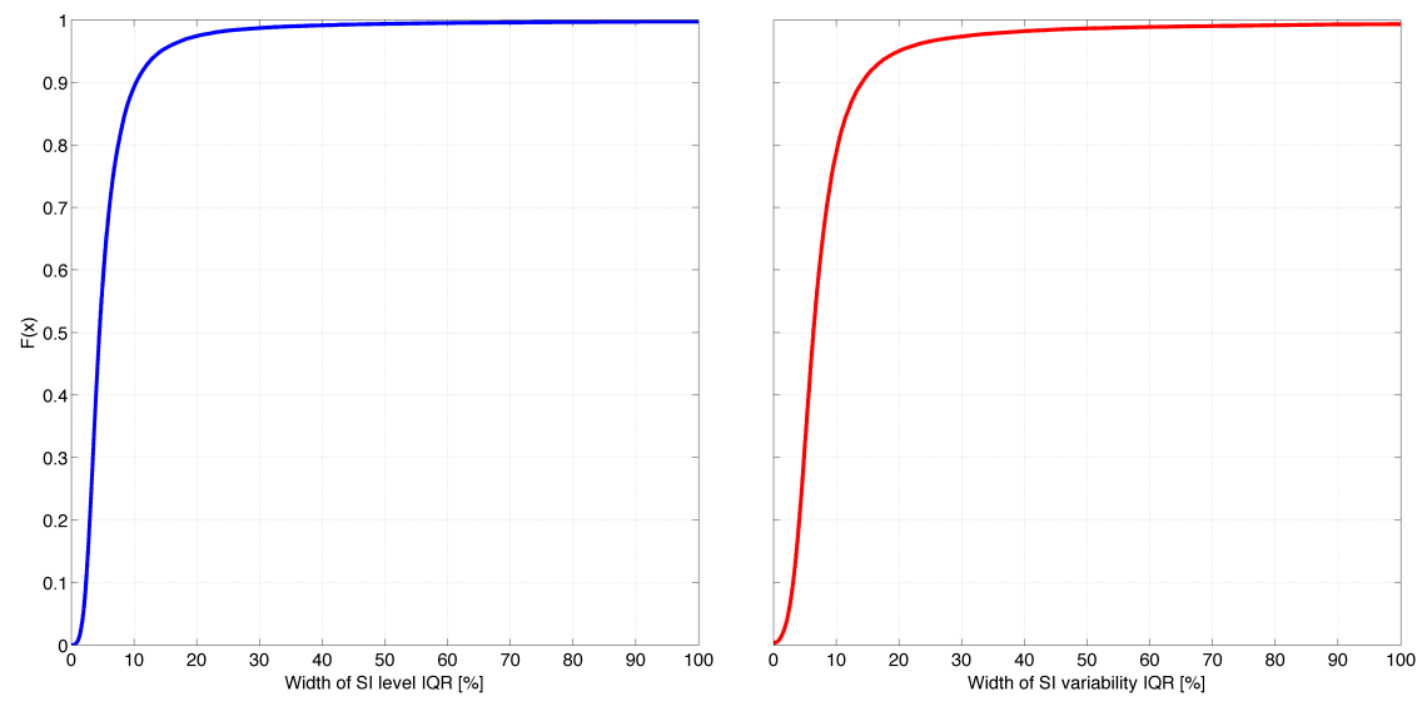

Figure 5. The impact of timing error on $S I$ level (left panel) and hour-to-hour variability (right panel), determined by Monte Carlo simulation.

These results show that typical timing errors in isolation have a relatively small impact on the level and variability of $S I$. With a median absolute difference between the simulated and actual measurement intervals of 9 minutes and using bolus insulin delivery, this result is not too surprising. Unlike infused insulin, bolus delivery ensures that the entire prescribed dose is always administered, regardless of the time between measurements. In addition, timing discrepancies only affect the later parts of the interstitial insulin profile, where concentrations are lowest and thus contribute least to the area under the curve used in fitting the SI parameter [10]. 


\subsection{Blood glucose sensor error}

Figure 6 shows the impact of BG sensor errors on SI level (left panel) and variability (right panel). Results from both error models are presented, with the solid lines indicating the model derived from clinical paired measurements (Figure 2) and the dashed line indicating the model derived from published data (Table 3). For $90 \%$ of hours, the IQR widths of SI level were less than $65 \%$ and $23 \%$ for the clinical and published models, respectively. Thus, for those 38,700 hours, half the simulations resulted in SI values within approximately $\pm 33 \%$ and $\pm 12 \%$ of the true value, in the case of a symmetrical distribution. Similarly, for variability, $90 \%$ of hours had IQR widths of hour-to-hour changes of less than $112 \%$ and $38 \%$, or $\pm 56 \%$ and $\pm 19 \%$ about the simulation median.

The variability induced in both $S I$ level and variability is significantly greater than that due to timing error in both cases. Additionally, the error model derived from clinical measurements causes greater variability than that from the published data.
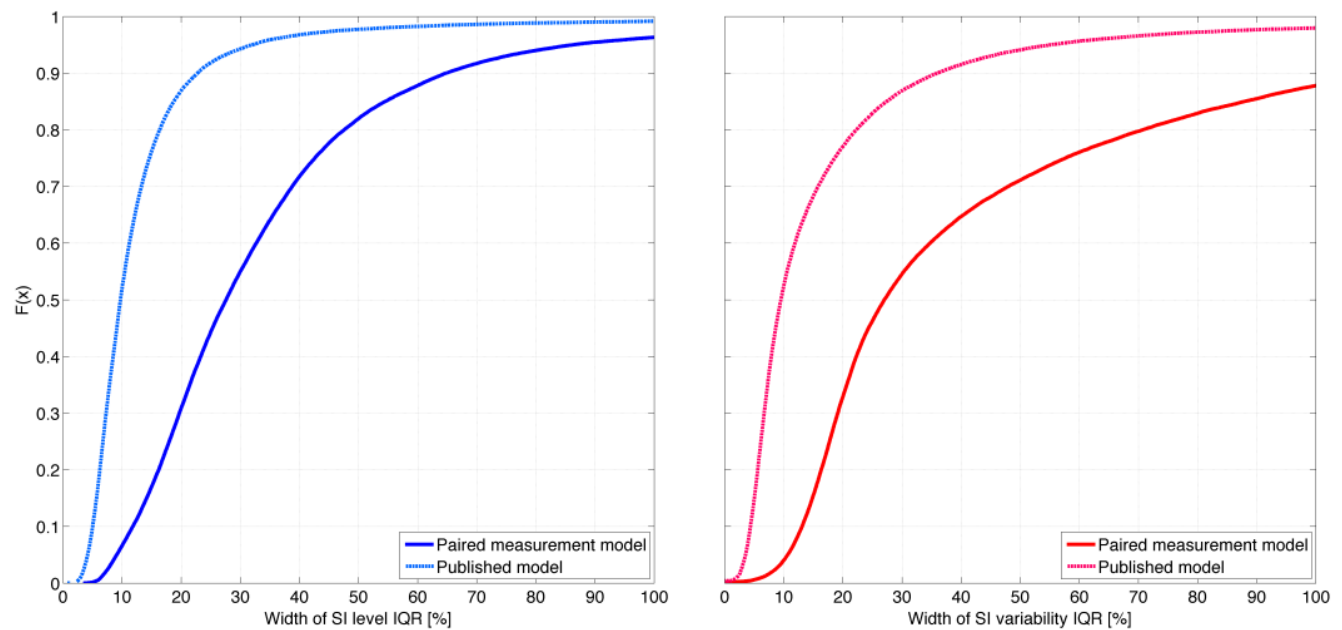

Figure 6. The impact of BG sensor error on SI level (left panel) and hour-to-hour variability (right panel), determined by Monte Carlo simulation.

\subsection{Combined measurement error}

Figure 7 shows the impact of the combined timing and BG sensor errors on SI level (left panel) and variability (right panel). The previous two sections have characterised the individual contributions of timing and sensor error. This 
analysis combines them, simulating errors seen in the real, clinical situation. The error model derived from paired clinical data was used as it represents the performance of the glucometer in an actual critical care environment.

For $90 \%$ of hours, the IQR width of SI level was less than $58 \%$. Thus, assuming a symmetrical distribution, half the simulations resulted in SI values within approximately $\pm 29 \%$ of the true value for these 38,700 hours. Similarly for variability, the $90^{\text {th }}$ percentile was $93 \%$, indicating that for half the simulations the hour-to-hour variability of SI was within $\pm 47 \%$.
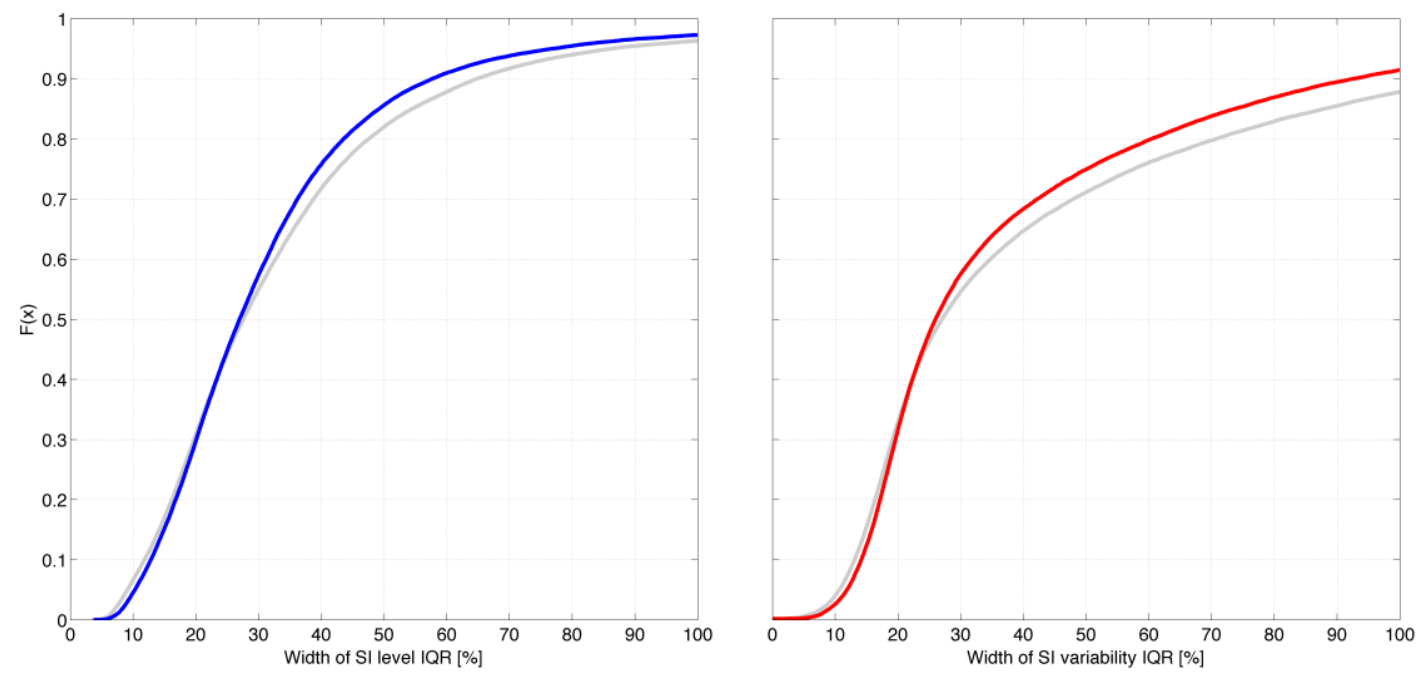

Figure 7. The impact of combined timing and BG sensor error on SI level (left panel) and hour-to-hour variability (right panel), determined by Monte Carlo simulation. The results of Figure 5 (paired measurement model) are shown as grey lines for comparison.

The results from Figure 6 are also shown on Figure 7 as grey lines. It is obvious that the two error sources are not additive and that the results are very similar. This similarity is possibly due to the effects of the two separate error sources cancelling each other out.

\subsection{Discussion}

The clinical impact during glycaemic control of changes to SI level induced by sensor and timing errors is potentially quite significant. Identified values of $S I$ were mostly within $29 \%$ of the true value when influenced by both sources of error. However, changes in SI greater than 20\% were seen with glucocorticoid treatment [15] and improving patient condition over the first 18 hours of ICU 
stay [16]. Thus, glycaemic control protocols that are designed to work with data from glucometers need to be robust to these errors and not be too aggressive in dosing insulin.

A second, potentially clinically significant, impact is on analytical use of $S I$ as a marker of injury or change in state. This analysis shows that identified shortterm changes in SI could be a result of measurement timing or sensor errors, rather than true physiological phenomena. Hence, using changes in SI level as a diagnostic must be done with caution, potentially looking at longer-term trends, where the effects of random errors may be cancelled by averaging over time.

In the context of the STAR protocol, the additional hour-to-hour variability caused by sensor and timing errors may be clinically significant. STAR uses a stochastic model of expected SI hour-to-hour variability to forecast the results of potential interventions and avoid hypoglycaemia. The stochastic model is derived from historical data measured with glucometers (Arkray SuperGlucocard II, Arkray Inc. Japan) and whole blood, and therefore includes the effects of BG sensor error that are likely to be of a similar magnitude to those observed in this study [4]. Specifically, the relatively large, $\pm 47 \%$, range of hourto-hour variability about the median caused by errors, suggests that a significant proportion of the expected variability may be a result these errors, rather than explicit physiological variability.

It is interesting to note that combining timing and sensor errors results in slightly less variability than the sensor error alone (Figure 7). This is likely a result of the effects of the two error sources cancelling each other out.

\subsection{Potential for reducing error and its impact on glycaemic control}

There is no effective way to remove these errors as they are random and apply equally to all patients. The only available option is to reduce the magnitude of the errors where possible and carefully manage their impact. The timing error distribution in Figure 3 shows that more than $85 \%$ of measurements are within 10 minutes of the scheduled time, which is a very good result in a busy ICU environment. 
In contrast, BG sensor errors can be reduced with better, more accurate, but likely more expensive equipment (for example, Abbott i-STAT, Nova StatStrip Glucose or a blood gas analyser). The Christchurch ICU is currently investigating several point-of-care blood glucose measurement devices for future clinical use. However, all measurement devices have some degree of uncertainty. In addition, other factors such as interfering substances and sampling procedure can also have a large impact on the accuracy of a measurement $[17,18]$. Thus, glucose control protocols must be robust to the impact of these errors.

The STAR protocol manages the variability induced in SI by considering the extreme percentiles of the stochastic model. By selecting interventions so that the forecast probability of the next blood glucose measurement being above or below the target band is just 5\% for each case, most of the induced variability is contained within the band, thus mitigating the clinical impact and potential risk of hypoglycaemia.

It is interesting to note the differences in accuracy of the glucometer between the published data and that obtained in a real, clinical setting. This study used data obtained from arterial blood samples collected by trained clinical staff and measured within 5 minutes, minimising the potential for additional error through device misuse $[17,18]$. However, there were still appreciable differences, and these may increase further in normal use, when clinical staff are not focused on a study protocol that is explicitly designed to reduce errors. 


\section{Conclusions}

The objective of this study was to assess and quantify the impact of typical timing and BG sensor errors on the level and variability of model-based SI. Specifically, the variability of level and the variability of SI hour-to-hour variability were investigated under the influence of these sources of error, both separately and combined. Measurement timing errors were shown to have a significant influence on the identified value of the SI parameter. The BG concentration errors of the Abbott Optium Xceed glucometer had a larger effect on $S I$ and dominated the combined analysis.

The clinical impact of errors on SI level during glycaemic control is likely to be significant and possibly detrimental to control performance. However, these changes are of similar magnitude to changes from physiological or therapy factors arising from the critical condition of the patients, which are already dealt

with by control protocols. Thus, glycaemic control protocols that are designed to work with data from glucometers need to be robust to these errors and not be too aggressive in dosing insulin. In contrast, the impact of errors on hour-to-hour SI variability is more pronounced and may impact the way the SI parameter is utilised for control and analysis.

This analysis indicated that for a given $S I$ level, variability induced by errors might dominate the IQR of the probability density function describing SI for the subsequent hour. In addition, using changes in SI level as a diagnostic must be done with caution, potentially looking at longer-term trends, where the effects of random errors may be cancelled by averaging over time.

Given the random nature of these errors, the only feasible method of mitigation is to use more accurate BG sensors and glycaemic control protocols that are robust to the impact of the errors. Understanding the effect of sensor and timing errors on SI allows their impact to be taken into account when using the parameter for control and analysis. 


\section{References}

[1] J. Lin, N. N. Razak, C. G. Pretty, A. Le Compte, P. Docherty, J. D. Parente, G. M. Shaw, C. E. Hann, and J. Geoffrey Chase, A physiological Intensive Control Insulin-Nutrition-Glucose (ICING) model validated in critically ill patients, Comput Methods Programs Biomed, vol. 102(2), pp. 192-205, May 2011.

[2] R. Hovorka, L. J. Chassin, M. Ellmerer, J. Plank, and M. E. Wilinska, A simulation model of glucose regulation in the critically ill, Physiol Meas, vol. 29(8), pp. 959-78, Aug 2008.

[3] Abbott, Optium Blood Glucose Test Strip datasheet, ed. UK: Abbott Diabetes Care Ltd, 2010.

[4] Arkray, Glucocard ${ }^{\mathrm{TM}}$ Test Strip 2 Data Sheet, ed. Japan: Arkray Inc., 2007.

[5] Roche, ACCU-CHEK Comfort Curve Test-strip packet insert, ed. USA: Roche Diagnostics Ltd, 2007.

[6] Roche, Evaluation Report of the Accu-Chek Comfort Curve Test Strip as a Plasma-like Test Strip, Roche Diagnostics, Mannheim, Germany, http://www.poc.roche.com/en US/pdf/Evaluation Report001.pdf.

[7] B. Solnica, J. W. Naskalski, and J. Sieradzki, Analytical performance of glucometers used for routine glucose self-monitoring of diabetic patients, Clin Chim Acta, vol. 331(1-2), pp. 29-35, May 2003.

[8] J. G. Chase, G. Shaw, A. Le Compte, T. Lonergan, M. Willacy, X. W. Wong, J. Lin, T. Lotz, D. Lee, et al., Implementation and evaluation of the SPRINT protocol for tight glycaemic control in critically ill patients: a clinical practice change, Crit Care, vol. 12(2), p. R49, 2008.

[9] E. Van Cauter, F. Mestrez, J. Sturis, and K. S. Polonsky, Estimation of insulin secretion rates from C-peptide levels. Comparison of individual and standard kinetic parameters for C-peptide clearance, Diabetes, vol. 41(3), pp. 368-77, Mar 1992.

[10] C. E. Hann, J. G. Chase, J. Lin, T. Lotz, C. V. Doran, and G. M. Shaw, Integralbased parameter identification for long-term dynamic verification of a glucose-insulin system model, Comput Methods Programs Biomed, vol. 77(3), pp. 259-270, Mar 2005.

[11] T. F. Lotz, J. G. Chase, K. A. McAuley, G. M. Shaw, X. W. Wong, J. Lin, A. Le Compte, C. E. Hann, and J. I. Mann, Monte Carlo analysis of a new modelbased method for insulin sensitivity testing, Comput Methods Programs Biomed, vol. 89(3), pp. 215-25, Mar 2008. 
[12] Abbott Diabetes Care, OptiumTest Strip Data Sheet, ed. UK: Abbott Diabetes Care, 2010.

[13] J. J. Mahoney and J. M. Ellison, Assessing glucose monitor performance--a standardized approach, Diabetes Technol Ther, vol. 9(6), pp. 545-52, Dec 2007.

[14] A. Evans, G. M. Shaw, A. Le Compte, C. S. Tan, L. Ward, J. Steel, C. G. Pretty, L. Pfeifer, S. Penning, et al., Pilot proof of concept clinical trials of Stochastic Targeted (STAR) glycemic control, Ann Intensive Care, vol. 1, p. $38,2011$.

[15] C. Pretty, J. G. Chase, J. Lin, G. M. Shaw, A. Le Compte, N. Razak, and J. D. Parente, Impact of glucocorticoids on insulin resistance in the critically ill, Computer Methods and Programs in Biomedicine, pp. 2-10, 2010.

[16] C. Pretty, A. Le Compte, J. G. Chase, G. M. Shaw, J. C. Preiser, S. Penning, and T. Desaive, Variability of insulin sensitivity during the first 4 days of critical illness: Implications for tight glycaemic control, Annals of Intensive Care, vol. In Press, 2012.

[17] R. M. Bergenstal, Evaluating the accuracy of modern glucose meters, Insulin, vol. 3, pp. 5-14, 2008.

[18] B. H. Ginsberg, Factors affecting blood glucose monitoring: sources of errors in measurement, J Diabetes Sci Technol, vol. 3(4), pp. 903-13, Jul 2009. 